\title{
Specific Antiproliferative Activity against Several Human Cancer Cells with Metabolites from Onygena corvina
}

\author{
Yukiko Ogawa $^{1}$, Fumihide Takano $^{2}$, Nobuo Yahagi ${ }^{3}$, Marie Yahagi ${ }^{3}$, \\ Yuki Kobayashi ${ }^{3}$ and Hidemitsu Kobayashi ${ }^{4}$
}

${ }^{1}$ Divisions of Infection Control and Prevention, ${ }^{4}$ Divisions of Microbiology, Department of

Pharmacy, Faculty of Pharmaceutical Science, Nagasaki International University, 2825-7

Huis Ten Bosch, Sasebo, Nagasaki 859-3298, Japan

${ }^{2}$ Division of Kampo Pharmaceutical Sciences, Nihon Pharmaceutical University, 10281

Komuro, Ina-machi, Kitaadachi-gun, Saitama 362-0806, Japan

${ }^{3}$ Yahagi Bio Institute, Mamurogawa-machi, Mogami-gun, Yamagata, 999-5604, Japan

*Corresponding author

\section{Keywords}

Onygena corvina,

Antitumor activity,

Cell specificity,

Medicinal plants,

Lung carcinoma

Article Info

Accepted:

12 December 2018

Available Online:

10 January 2019

\section{A B S T R A C T}

We succeeded in large volume artificial cultivation of Onygena corvina. The appearance (shape, color, and size) of fruiting bodies of cultured bacteria was almost the same as that of wild one. We screened their metabolites for antiproliferative activities against nine human cancer cells in vitro. Culture filtrates (metabolite-containing media) of $O$. corvina were lyophilized and resuspended in phosphate buffer to investigate them in vitro. As a result, these metabolites effectively inhibited the growth of some cancer cells in a concentration-dependent manner. In particular, the effect on lung carcinoma Hara cells was remarkable, but there was almost no effect on hepatoma HepG2 cells. In addition, almost no effect was exerted on three normal cells [human hepatocyte (NHH), human mammary epithelial cells (NHME), and human epidermal melanocytes (NHEM)] at the same concentration. Therefore, it was revealed that the human cell proliferation inhibitory effect of the $O$. corvina metabolites greatly differs in susceptibility depending on the type of cancer cell under the concentration range not affecting normal cells. In vivo experiments demonstrated that the oral administration of the $O$. corvina metabolites (OC-FD) decreased the tumor growth compared with untreated mice. The OCT extract obtained by extracting ethanol had the same anti-tumor activity as the OC-FD, but this activity was weaker than with OC-FD. On the other hand, when the same experiment was performed on OCT with 2.O.C.11 and 1.O.C.5, respectively, for the EtOAc soluble fraction and water layer, this demonstrated stronger inhibitory activity than when only the OC-FD or OCT was administered. These results revealed these active components may be both low-molecular weight with low polarity (EtOAc soluble) and high-molecular weight, such as a polysaccharide or protein (water soluble). These findings suggest that the unknown compounds in the metabolite-containing media from cultures of $O$. corvina are potential lead compounds for developing anticancer drugs with extremely mild side effects. 


\section{Introduction}

Treatment strategies have undergone significant changes in the $21^{\text {st }}$ Century. Surgical treatment is, even now, an essential part of solid cancer treatment, but treatment policy has shifted from the extended operations used thus far to functional preservation strategies that focus on the patient QOL, and treatment methods that highly value the informed consent of the patient (Tian et al., 2018). With chemotherapy, there is high-dose chemotherapy through targeting therapy that uses DDS (Nakamura et al., 2018), as well as bone marrow transplantation. For radiotherapy, treatment takes place using new methods such as the $\mathrm{X}$ knife, etc. (Alster et al., 2018), and a new method for which there are high expectations in the $21^{\text {st }}$ Century is cancer gene therapy. Under particular focus is "precision therapy," which is starting to be introduced for lung cancer therapy to provide individual treatment for different types of cancer on an individual level (Kehl et al., 2018, Lu et al., 2018).

Genus Cordyceps is a type of natural caterpillar fungus, in which caterpillar fungus infests living insects (Sato et al., 2002), forms hypha nuclei within the body, and seasonally extends and develops from the head section and joints of the insect to the fruiting body (Takano et al., 1996, Yahagi et al., 1999, Yahagi et al., 2004). Approximately 350 or more types of genus cordyceps have been discovered throughout the world and, since ancient times in China (Zhu et al., 1998), it has been known to be a nourishing tonic, and considered effective in increasing longevity, providing anti-oxidant effect, and increasing immunological effects (Jeong et al., 2013, Xiao et al., 2017). In Japan, 300-400 types of caterpillar fungus infesting a large number of insects, such as cicada and silkworms (Chen $e t$ al., 2002), have been discovered. These have been shown to have a number of benefits, such as cancer cell inhibitory effects (Bok et al., 1999, Chen et al., 2013, Ogawa et al., 2014), anti-oxidant effects, and immunostimulatory effects (Choi et al., 2004, Paterson et al., 2008, Bhatti et al., 2013, Cheng et al., 2013). The caterpillar fungus in silkworms promises to have a repairing effect on the hippocampus for Alzheimer's-type dementia (Tsushima et al., 2010). Caterpillar fungus works to strengthen the lungs and kidneys and is used as a core treatment for emphysema and asthma in China, in combination with other herbal medicines (Wang et al., 2016). $\beta$-glucan is included in extremely high quantities in caterpillar fungus (Smiderle et al., 2014), 17 times the amount contained in agaricus mushrooms and 170 times that of normal fungi. It has a rich composition rate of nutritional elements, including minerals such as zinc or selenium, as well as natural amino acids and proteins. It also includes cordycepin and antioxidant enzymes (S.O.D), and is gaining attention for its anticancer effects (Choi et al., 2012, Choi et al., 2013, Shao et al., 2016).

On the other hand, Onygena corvina (feather stalkball) and Onygena equinea (horn stalkball), both species of the fungal genus Onygena in Onygenaceae family, can live as saprophytes on feathers, hooves, horn, and hair (Lange et al., 1975) which is a rare bacterium in the world, is a fungus that infests the bones of animals. Whereas Japanese caterpillar fungus is hosted by insects, Onygena is hosted in the bones, vomit, and excreta of mammals, and therefore promises to provide more powerful enzyme "physiologically active substances." However, examples of its occurrence are extremely rare, and there are virtually no reports of it being picked in the wild. This, in turn, means that there are few examples of academic papers or experiments, and there are many fields which are yet unknown. On this occasion, we have 
been successful in artificially cultivating it for the first time in Japan. We have evaluated the anti-tumor effects of the picked and cultivated Onygena and metabolites from Onygena corvina by administering four types of Onygena extract using Sarcoma-180 solid sarcoma tumor model mice with the aim of exploring its anti-tumor activity elements. Additionally, we performed screening in relation to anti-growth inhibitory activities in several types of human cancer cells within the culture metabolite.

\section{Materials and Methods}

\section{Genus Onygena fungal mycelial cultibation}

Parasitic mushroom, Onygena corvina was harvested from skull of a weasel at Sakekawamura (Mogami-Gun, Yamagata Prefecture, Japan). The photograph of O.carvina in the natural field was shown in figure 1A. Onygena picked in the wild and its conidium was first injected into agar. After cultivation, the cultivated mushroom bed was moved to a liquid culture including yeast and cultured for a fixed period of time.

The bacteria were removed when a fixed number of conidophore bundles formed in the liquid medium, and the liquid medium was filtered and freeze-dried (OC culture medium extract: OC-FD). In addition, the OC extract was infiltrated with ethanol and, after concentrating the ethanol-soluble fraction, an extract was obtained (OCT). $150 \mathrm{~mL}$ of water was added to the OCT extract and a separating funnel was used to distribute $150 \mathrm{~mL}$ of ethyl acetate (EtOAc).

The distribution operation was repeated three times with $150 \mathrm{~mL}$ of the EtOAc and water, respectively. The EtOAc soluble fraction and water fraction obtained from this were vacuum-concentrated or freeze-dried, respectively, to make 2.O.C.11 (EtOAc soluble) and 1.O.C.5 (water soluble).

\section{Chemicals}

Cell Counting Kit-8 including 2-(2-methoxy4-nitrophenyl)-3-(4-nitrophenyl)-5-(2,4disulfophhenyl)-2H-tetrazolium, monosodium salt (WST-8) was from Dojindo Co., Kumamoto, Japan. RPMI 1640 medium was from Nissui Pharmaceutical Co., Tokyo, Japan. Fetal calf serum (FCS) was from Life Technologies Co., Carlsbad, CA, USA. Penicillin- streptomycin was from Roche Diagnostics K. K., Tokyo, Japan. Trypan blue solution was from Nacalai Tesque, Inc., Kyoto, Japan. Aphidicolin was purchased from Wako Pure Chemical Industries, Osaka, Japan.

\section{Cells}

Human breast cancer MCF-7 and MDA-MB231 cells, human gastric cancer Kato III cells, human colon adenocarcnoma Colo201 and Caco2 cells, human hepatoma HepG2 cells, human lung squamous cell carcinoma Hara cells, human promonocytic leukemia U937 cells and human malignant melanoma GAK cells were obtained from the JCRB Cell Bank (National Institute of Biomedical Innovation, Health and Nutrition) and ATCC Cell Biology Collection, respectively.

Normal human hepatocytes (NHH), normal human mammary epithelial cells (NHME), Normal human epidermal melanocytes (NHEM) were purchased from Promo Cell Co. (Heidelberg, Germany). MCF-7, Caco-2 and HepG2 were maintained in E-MEM and D-MEM, respectively. KATO III, Colo201, U937 and Hara cells were grown in RPMI1640 medium. All cell cultures were supplemented with heat-inactivated fetal calf serum $10 \%(\mathrm{v} / \mathrm{v})$, penicillin $(100 \mathrm{IU} / \mathrm{ml})$, and streptomycin $(100 \mu \mathrm{g} / \mathrm{mL})$ at $37{ }^{\circ} \mathrm{C}$ in an atmosphere of $95 \%$ air/ $5 \% \mathrm{CO}_{2}$. MDA-MB231 and GAK were maintained in L-15 medium and Ham's F12 medium were supplemented with heat-inactivated fetal calf 
serum $20 \%(\mathrm{v} / \mathrm{v})$, penicillin $(100 \mathrm{IU} / \mathrm{ml})$, and streptomycin $(100 \square \mathrm{g} / \mathrm{ml})$ at $37{ }^{\circ} \mathrm{C}$ in an atmosphere of $95 \%$ air/ $5 \% \mathrm{CO}_{2}$.

\section{Creation of a cancer bearing model}

Solid sarcoma cell Sarcoma-180 (S-180) was transplanted at $0.05 \mathrm{~mL} / \mathrm{animal}$ to the right lower abdomen limb root of ICR mice acclimatized and bred over one week to make a concentration of $1 \times 10^{6}$ cells $/ \mathrm{mL}$.

After transplantation, the body weight and tumor volume were periodically measured, the tumor mass was excised, and its weight was measured at the end of the experiment. The tumor volume was calculated from the product of the major and minor axis of the tumor, under the presumption that it is the same as the density of the cancel cells.

The four types of Onygena prepared to make concentrations of $300 \mathrm{mg} / \mathrm{mL} \quad(0.01 \mathrm{~mL} / \mathrm{g}$ body weight in saline) were administered orally from the start date of injecting S-180 once per day for 30 days.

\section{Cell viability and cytotoxic assay}

Cells $\left(2 \times 10^{4}\right.$ cels/well $)$ were seeded into a 96well flat-bottom plate and treated with various concentrations $(0-2500 \mu \mathrm{g} / \mathrm{mL})$ of OC-FD for $48 \mathrm{~h}$ at $37{ }^{\circ} \mathrm{C}$ in an atmosphere of $95 \%$ air and $5 \% \mathrm{CO}_{2}$. Cytotoxic activity and cell viability and cell growth were evaluated by trypan blue $(0.5 \%(\mathrm{w} / \mathrm{v}))$ exclusion and by the WST-8 assay, respectively. The reduction in proportion of living cells was assayed by measurement of absorbance at $450 \mathrm{~nm}$ (reference, $600 \mathrm{~nm}$ ) using the GloMax Multi Detection System.

\section{Statistical analysis}

The results of experiments are presented as mean \pm standard error (SE). Differences in means were evaluated by two-tailed Student's $t$-test with $P$ values $<0.05$ considered to be statistically significant.

\section{Results and Discussion}

\section{Culture of $O$. corvina and preparation of secondary metabolites}

The Onygena fungus is in the Ascomycota group and is often broken down in a saprophytic manner in animal carcasses, but there are those that infest and become pathogens in animals, and those that form symbiosis and form mycorrhiza in the Ericaceaee family of plants (Doveri et al., 2012).

Ecologically, it is a mixture of a wide variety of groups. Additionally, because some of these have strong keratin decomposition abilities, even organisms that are suited to processing industrial waste such as bird feathers have gained attention recently (Gupta et al., 2013, Sharma et al., 2011). However, there are very few examples demonstrating a scientific basis for its effectiveness.

We successfully developed a stable Chu-Soh culture system that has a high level of safety (Yahagi et al., 1999). It also permits the efficient formation of sexual fruiting bodies on the culture medium that are comparable with those that develop in the wild, and allow the production of different lots with the same efficacy.

Using this artificial liquid medium, we were able to culture samples in large quantities (Yahagi et al., 2004). Figure 1B shows the fruiting bodies developed from $O$. corvina on agar. The fruiting body had similar form, size, and color compared to wild $O$. corvina. The metabolite-containing media obtained from this culture system were filtered through a membrane filter and freeze-dried for use in subsequent experiments. 
Table.1 Influence of Onygena corvina on mouse body weight changes

\begin{tabular}{|l|c|c|c|c|c|c|c|}
\hline & \multicolumn{7}{|c|}{ Body weight (g) } \\
\cline { 2 - 8 } & Day 0 & Day 5 & Day 10 & Day 15 & Day 20 & Day 325 & Day 30 \\
\hline Control & 20.1 & 20.7 & 21.0 & 21.7 & 23.5 & 25.2 & 26.9 \\
\hline OC-FD i.p. & 20.2 & 20.6 & 21.1 & 21.6 & 23.8 & 25.4 & 26.3 \\
\hline OC-FD p.o & 19.6 & 20.3 & 20.9 & 21.3 & 22.6 & 24.6 & 26.8 \\
\hline OC-FD i.v. & 20.1 & 20.8 & 31.1 & 21.8 & 23.3 & 25.0 & 27.1 \\
\hline
\end{tabular}

OC-FD were orally, intraperitoneally and intravenously administered to mice, respectively, and the changes in weight of the mice over 4-weeks were measured.

Table.2 Antitumor effect of Onygena corvina on tumor bearing model

\begin{tabular}{|c|c|c|c|c|c|}
\hline & \multicolumn{3}{|c|}{ Body weight (g) } & \multirow{2}{*}{$\begin{array}{l}\text { Av.tumor } \\
\text { weight (g) }\end{array}$} & \multirow{2}{*}{$\begin{array}{l}\text { Inhibition } \\
\text { ratio (\%) }\end{array}$} \\
\hline & Day 0 & Day 16 & Day 35 & & \\
\hline Control & 25.6 & 29.6 & 42.1 & 7.43 & \\
\hline OC-FD & 25.1 & 29 & 39.7 & 5.84 & 21.3 \\
\hline OCT & 25.1 & 29.1 & 36.7 & 4.7 & 36.7 \\
\hline 2.O.C. 11 & 25.5 & 28.7 & 36.5 & 3.24 & 56.4 \\
\hline 1.O.C. 5 & 25.3 & 29.2 & 37.6 & 2.75 & 62.9 \\
\hline
\end{tabular}

S-180 was transplanted to the ICR mice. After transplantation, the body weight and tumor volume were periodically measured, the tumor mass was excised, and its weight was measured at the end of the experiment. The tumor volume was calculated from the product of the major and minor axis of the tumor, under the presumption that it is the same as the density of the cancel cells. The four types of Onygena prepared to make concentrations of 300 $\mathrm{mg} / \mathrm{mL}(0.01 \mathrm{~mL} / \mathrm{g}$ body weight in saline) were administered orally from the start date of injecting S-180 once per day for 30 days. Data are the means of triplicate assay mean \pm SE.

Fig.1 Artificial culture and natural products of Onygena corvina
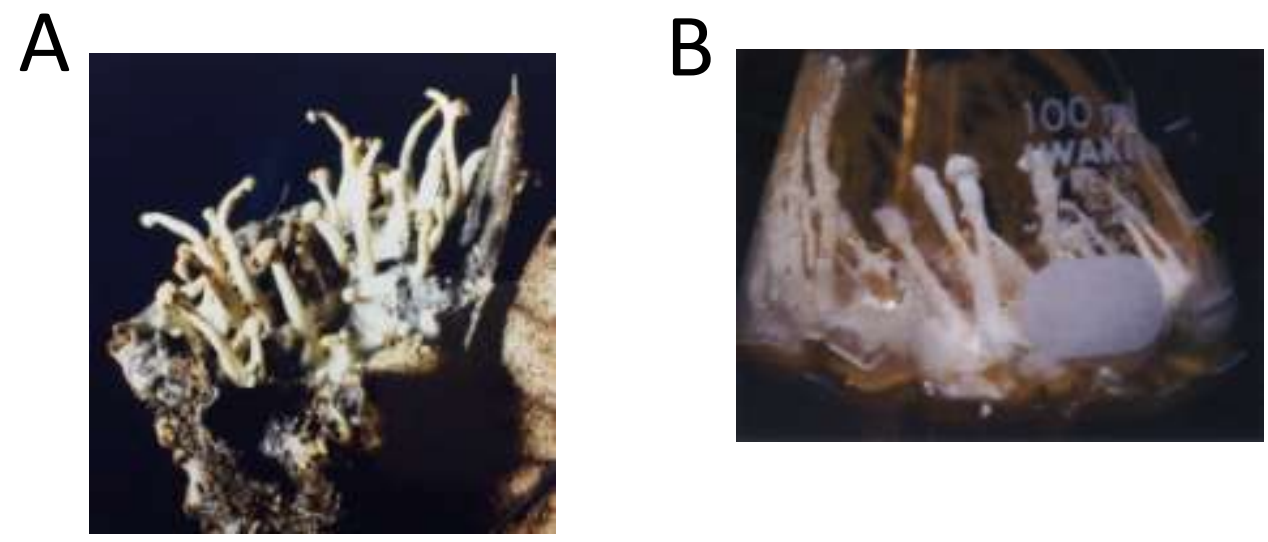

(A) Specimens of Onygena corvina. in natural field

(B) Fruiting bodies produced by Onygena corvina. in culture medium. 
Fig.2 Inhibitory effect of secondary metabolites of Onygena corvine (OC-FD) on the growth of human tumor cells

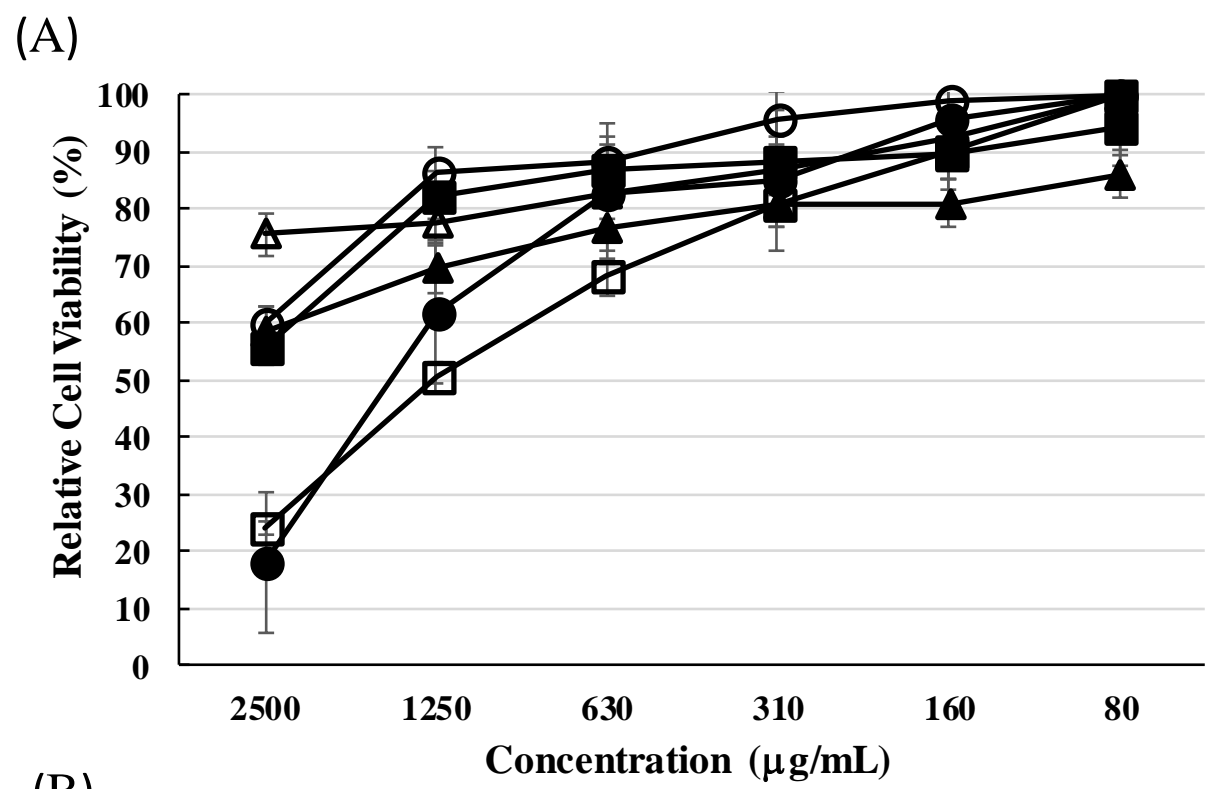

(B)

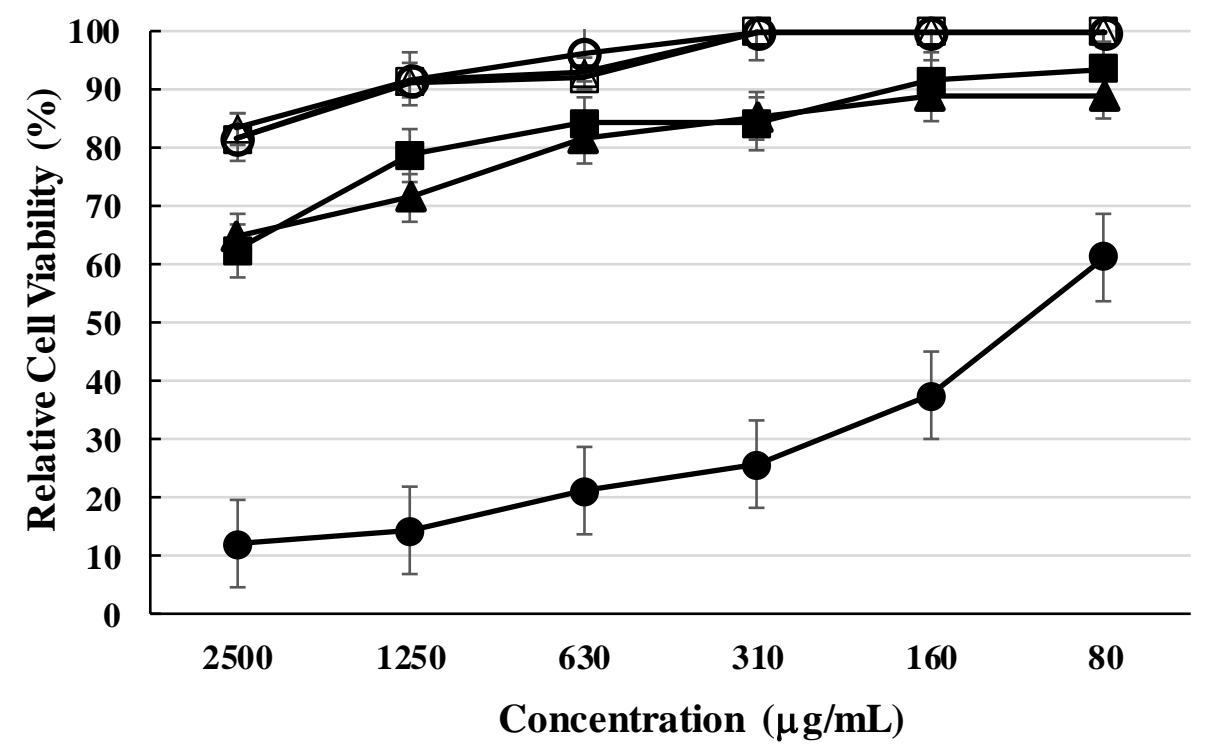

Cells were treated with OC-FD [1250-80 $\mu \mathrm{g} / \mathrm{mL}]$ for $48 \mathrm{~h}$. Then cell viability was determined by WST-8 assay and trypan blue dye exclusion assay. Error bars: SE from three different cell preparations assayed individually. (A)

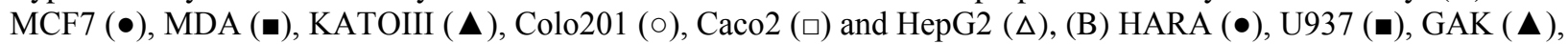
$\operatorname{NHH}(\circ), \operatorname{NHME}(\square)$ and $\operatorname{NHEM}(\Delta)$

Antiproliferative activity of $O$. corvina secondary metabolites in human cancer cells

We succeeded in large volume artificial cultivation of $O$. corvina. To investigate the effect of $O$. corvina secondary metabolites (OC-FD) on cell proliferation in various types of human cancer cells [human breast cancer (MCF7, MDA), gastric cancer (Kato III), 
colon adenocarcinoma (Colo201, Caco2), leukemia (U937), lung carcinoma (Hara), hepatoma (HepG2), human malignant melanoma (GAK)], and three normal cells [human hepatocyte (NHH), human mammary epithelial cells (NHME), human epidermal melanocytes (NHEM)]. OC-FD significantly suppressed cell growth in MCF7, Caco2 and Hara cells and extremely strong growth inhibition activity was especially seen for lung carcinoma Hara cells. At this concentration, there was hardly any effect on normal cells (Fig. 2). The antiproliferative metabolites obtained from OC-FD were highly specific to human cancer cell lines. These findings suggest that the unknown compounds in the metabolite-containing media from cultures of these mushrooms are potential lead compounds for developing anticancer drugs with extremely mild side effects.

\section{Influence of $O$. corvina on mouse body} weight changes

Antiproliferative activities of culture filtrates from $O$. corvina are rarely observed in normal human skin fibroblasts at a range of concentration where the effects are observed in cancer cells. Moreover, in our experiments on acute and chronic toxicities in mice, there was not a large difference in weight gain between the control and test groups (Table 1), and no abnormalities in isolated organs were observed in the test group (data not shown). Taking into consideration data from the above, some ingredients in metabolitecontaining media of $O$. corvina are expected to be potential novel lead compounds for developing anticancer drugs with extremely mild side effects.

\section{Antitumor effect of $O$. corvina on tumor bearing model}

As shown in Table 2, in control mice for which the S-180 intake sample was not administered, solid cancer was formed and, as time passed, the mass and volume of the tumor increased. In contrast, in the mice for which OC extract (OC-FD) as administered at doses of $300 \mathrm{mg} / \mathrm{kg} / \mathrm{day}$, the volume and mass of the tumors increasing over time was controlled. The OCT extract obtained by extracting ethanol had the same anti-tumor activity as the OC-FD, but this activity was weaker than with OC-FD. On the other hand, when the same experiment was performed on OCT with 2.O.C.11 and 1.O.C.5, respectively, for the EtOAc soluble fraction and water layer, this demonstrated stronger inhibitory activity than when only the OC-FD or OCT was administered. In the diagram comparing the weight of the solid cancer excised at the end of the experiment, the strongest activity was shown in the OC fraction (2.O.C.11 [EtOAc soluble] and 1.O.C.5 [water soluble]). From the above results, oral administration of the $O$. corvina cultured metabolite solution OC clearly demonstrated anti-tumor activity, and it was shown that this active element was not an element that was soluble in ethanol and had comparatively high water solubility. Additionally, in the ethyl acetate (EtOAc) soluble fraction 2.O.C.11 and water layer 1.0.C.5 obtained from liquid-liquid distribution from OCT, stronger activity was recognized than in single administrations of OC, which suggests that this active components may be both low-molecular weight with low polarity (EtOAc soluble) or high-molecular weight, such as a polysaccharide or protein (water soluble). Moving forward, investigating what kind of elements are contained in 1.O.C.5 fractions that demonstrate particularly strong activity and reevaluating the anti-tumor activity of model animals will provide evidence of the effectiveness of $O$. corvina in battling cancer.

To the best of our knowledge, the present study was the first to demonstrate that culture 
filtrates (metabolite-containing media) of $O$. corvina may inhibit the viability of breast cancer cells, colon carcinoma cells and lung carcinoma cells in vitro, and sarcoma solid tumor cells in vivo. The metabolite-containing medium harvested from $O$. corvina is considered to contain many substances including polysaccharides. In order to identify the bioactive forms of $O$. corvina, we are currently working towards isolated purification of the active fractions using highperformance liquid chromatography after fractioning by various organic solvents and determining the chemical structures by focusing on instrumental analysis such as NMR method, and mass spectrometry. On the other hand, experiments to clarify anti-cancer activity mechanisms of the active fractions which have been purified by chromatography, and toxicity tests on mice have carried out accurately, and publication of the details are planned for the next thesis. In the near future, $O$. corvina is expected to become a valuable resource for combined therapy with an anticancer drug, at a dosage which does not show side effects, and a leading compound for novel anticancer agents.

\section{References}

Alster, P., Koziorowski, D.M., Za Bek, M., Dzierzęcki, S., Ma Dry, J., DuszyńskaWa, S. K., Grygarowicz, H., Zielonko, J., Królicki, L., Friedman, A., 2018. Making a Difference-Positive Effect of Unilateral VIM Gamma Knife Thalamotomy in the Therapy of Tremor in Fragile X-Associated Tremor/Ataxia Syndrome (FXTAS). Front. Neurol., 9, 512. doi:10.3389/fneur.2018.00512.

Bok, J.W., Lermer, L., Chilton, J., Klingeman, H.G., Towers, G.H., 1999. Antitumor sterols from the mycelia of Cordyceps sinensis. Phytochemistry, 51, 891-898.

Bhatti, M.T., Freedman, S.M., Mahmoud,
T.H., 2013. Fingolimod Therapy and Mocular Hemorrhage. J. neuroophthalmol., 33(4), 370-372.

Chen, R., Ichida, M., 2002. Infection of the silkworm, Bombyx mori, with Cordyceps militaris. J. Inect Biotech. Sericol., 71, 61-63.

Chen, Y.H., Wang, J.Y., Pan, B.S., Mu, Y.F., Lai, M.S., So, E.C., Wong, T.S., Huang, B.M., 2013. Cordycepin enhances cisplatin apoptotic effect through caspase/MAPK pathways in human head and neck tumor cells. Onco. Tagets Ther., 25, 983-998.

Choi, G.Y., Choi, B.T., Jeog, Y.H., Jeong, Y.K, 2013. Apoptosis induction of human prostate carcinoma cells by cordycepin through reactive oxygen species-mediated mitochondrial death pathway. J. Oncology, 42, 1036-1044.

Gupta, R., Sharma, R., Beg, Q.K., 2013. Revisiting microbial keratinases: next generation proteases for sustainable biotechnology. Crit., Rev., Biotechnol., 33, 216-228.

Cheng, Y.W., Chen, Y.I., Tzeng, C.Y., Chang, C.H., Lee, Y.C., Chen, H.C., Tsai, C.C., Hsu, T.H., Lai, Y.K., Chang, S.L., 2013. Aqueous extracts of Cordyceps militaris (Ascomycetes) lower the levels of plasma glucose by activating the cholinergic nerve in streptozotocin-induced diabetic rats. Int J Med Mushrooms, 15, 277-286.

Choi, S.B., Park, C.H., Choi, M.K., Jun, D.W., Park, S., 2004. Improvement of insulin resistance and insulin secretion by water extracts of Cordyceps militaris, Phellinus linteus, and Paecilomyces tenuipes in $90 \%$ pancreatectomized rats. Biosci. Biotechnol. Biochem., 68, 2257-2264.

Doveri, F., Pecchia, S., Vergara, M., Sarrocco, S., Vannacci, G., 2012. A comparative study of Neogymnomyces virgineus, a new keratinolytic species 
from dung, and its relationships with the Onygenales. Fungal Divers, 52, 13-34.

Jeong, M.H., Lee, C.M., Lee, S.W., Seo, S.Y., Kang, B.W., Jeong, Y.K., Choi, Y.J., Yang, K.M., Jo, W.S., 2013. Cordycepin-enriched Cordyceps militaris induces immunomodulation and tumor growth delay in mousederived breast cancer. Oncol. Rep., 30, 1996-2002.

Jeong, J.W., Jin, C.Y., Park, C., Han, M.H., Kim, G.Y., Moon, S.K., Kim, C.G., Jeong, Y.K., Kim, W.J., Lee, J.D., Choi, Y.H., 2012. Inhibition of migration and invasion of $\mathrm{LNCaP}$ human prostate carcinoma cells by cordycepin through inactivation of Akt. Int. J.Oncol., 40, 1697-1704.

Kehl, K.L., Lathan, C.S., Johnson, B.E., Schraq, D., 2018. Race, Poverty, and Initial Implementation of Precision Medicine for Lung Cancer. J. Natl. Cancer Inst., doi:10.1093/jnci/djy202.

Lange, M., Hora, F.B.(eds), 1975. Collins guide to mushrooms \& toadstool. Collins, London.

Lu, J., Han, B., 2018. Liquid Biopsy Promotes Non-Small Cell Lung Cancer Precision Therapy, Technol Cancer Res.Treat., 17, 1533033818801809

Nakamura, T., 2018. Development of a Nano DDS for Cancer Immunotherapy Based on Llipid Nanoparticles. Yakugaku Zasshi, 138, 1443-1449.

Ogawa, Y., Yahagi, N., Yahagi, R., Kobayashi, H., 2014. Specific antiproliferative activity against human cancer cells with metabolites from several species related to the genus Cordyceps, Int. J. Curr. Microbiol. App. Sci., 3(5), 607-617.

Paterson, R.R.M., 2008. Cordyceps-A traditional Chinese medicine and another fungal therapeutic biofactory. Phytochemistry. 69, 1469-1495.

Sharma, R., Murty, N. A., Gupta, R., 2011.
Molecular characterization of Nterminal pro-sequence of keratinase ker $P$ from Pseudomonas aeruginosa: identification of region with chaperone activity. Appl. Biochem. Biotechnol., 165, 892-901.

Sato, H., Shimizu, M., 2002. Storomata production for Cordyceps militaris (Clavicipitales: Clavicipitaceae) by infection of hyphal bodies to alternative host insects. Appl. Entomol. Zool., 37, 85-92.

Shao, L.W., Huang, L.H., Yan, S., Jin, J.D., Ren, S.Y., 2016. Cordycepin induces apoptosis in human liver cancer HepG2 cells through extrinsic and intrinsic signaling pathways. Oncol. Lett., 12(2), 995-1000.

Smiderle, F.R., Baggio, C.H., Borato, D.G., Santana-Filho, A.P., Sassaki, G.L., Iacomini, M., Van Griensven Leo, J.L.D., 2014 . Antiinflammatory properties of the medicinal mushroom Cordyceps militaris might be related to its linear $(1 \rightarrow 3)-\beta$-D-glucan. PLOS ONE, $9(10)$,

doi.org/10.1371/journal.pone.01102 66

Takano, F., Kikuchi, Y., Fushiya, S., Hojo, H., Nozoe, S., Yahagi, N., Kondo, Y., 1996. The culture fluid of Isaira japonica yasuda augments anti-sheep red blood cell antibody response in mice. Biol. Pharm. Bull. 19, 641-643.

Tian, J.Y., Ying, Y.L., Xin, H., Gen, H.Di., 2018. Survival following breastconserving therapy is equal to that following mastectomy in young women with early-stage invasive lobular carcinoma. Eur. J. Sur. Oncol., 44(11), 1703-1707.

Tsushima, M., Yamamoto, K., Goryou, M., Suzuki, F. and Suzuki, K., 2010. Hotwater extract of Paecilomyces tenuipes from the silkworm pupae improves D- 
galactose-induced brain aging in mice. J. Insect Biotec. Sericol., 79, 45-51.

Wang, N., Li J., Huanq, X., Chen, W., Chen, Y., 2016. Herbal Medicine Cordyceps sinensis Improves Health-Related Quality of Life in Moderate-to-Severe Asthma. Evid. Based Complement. Alternat. Med., 2016, Article ID 6134593,8 pages.

Yahagi, N., Komatsu, M., Hiramatsu, M., SHI, H., Yahagi, R., Kobayashi, H., Kamada, H., Takano, F., Fushiya, S., 1999. Radical Scavenging Activities of Condensed Culture Medium of Isaria japonica Yasuda and Hot water Extract of Fomes fomentarius (L.:Fr) Kicrx. Nat. Med., 53, 319-323.

Yahagi, N., Yahagi, R., Takano, F., Fushiya,
S., Tanaka, T., Tanaka, K., Ohta, T., 2004. Growth of ascocarps from cultured Cordyceps militaris (L.:Fr.) Fr. and Cordyceps formicarum Kobayasi in an agar medium, Kingakukai kaiho, 45, $15-19$.

Xiao, Y., Huanq, Q., Zhenq, Z., Quan, H., Liu, S., 2017. Construction of a Cordyceps sinensis exopolysaccharideconjugated selenium nanoparticles and enhancement of their antioxidant activities. Int. J. Biol. Macromol., 99, 483-491.

Zhu, J.S., Halpern, G.M., Jones, K., 1998. The Scientific Rediscovery of an Ancient Chinese Herbal Medicine: Cordyceps sinensis Part I, J. Altern. Complement. Med., 4: 289-303.

\section{How to cite this article:}

Yukiko Ogawa, Fumihide Takano, Nobuo Yahagi, Marie Yahagi, Yuki Kobayashi and Hidemitsu Kobayashi. 2019. Specific Antiproliferative Activity against Several Human Cancer Cells with Metabolites from Onygena corvine. Int.J.Curr.Microbiol.App.Sci. 8(01): 1659-1668. doi: https://doi.org/10.20546/ijcmas.2019.801.174 